\title{
Radon as a Early Warning Tool in Tectonic Monitoring Environments Analyzing Data Behavior
}

\author{
Research Article \\ V. I. Outkin ${ }^{1}$, Pushan Kumar Dutta ${ }^{2}$, O. P. Mishra ${ }^{3}$, M. K.Naskar ${ }^{2}$, I. A. Kozlova ${ }^{1}$, A. K. Yurkov ${ }^{1}$
}

1 Institute of geophysics, Ekaterinburg, Amundsen str.100,620016, Yekaterinburg/GMT+6, Russia

2 Electronics and Tele-Communication Dept. Jadavpur University, Kolkata, West Bengal 700032/GMT+5:30, India

3 SAARC Disaster Management Centre (SDMC), IIPA Campus, Ring Road Delhi, Delhi-110002/GMT+5:30, India and Geo-seismology Division, Geological Survey of India (CHQ), Kolkata

\begin{abstract}
:
The proposed work describes research of the behavior of radon for volume activity of radon (VAR) excreted from the array. Radon migration and its selection from the array depends on the porosity, permeability and fractures in the array. A drastic change in the strength of an array and reset the elastic stresses by external forces (earthquake) occurs when certain changes in the structure of the array as the compressive and tensile strength of the array.
\end{abstract}

Keywords:

Volume activity of radon $(\mathrm{VAR}) \cdot$ changes in the VAR $\bullet$ the critical state of the array $\bullet$ the external impact of trigger to earthquake $\cdot$ remote sensing (C) Versita sp. z o.o.

Received 28-05-2013; accepted 12-07-2013

\section{Introduction}

Research on geochemical precursory algorithms aimed at defining quantitative relations between seismogenic parameters and endogenetic components shows that despite of development of the seismometric equipment, methods of processing of seismic signals, development of seismometric complexes etc., the problem of the forecast of tectonic earthquakes (especially short-term) remains unsolved as passive seismological signals not always remit deterministic evidence for preparing event with speed of occurrence of seismological signals. Any anomalous state is concurrent with a pre-slip for a fractured medium. In a fractured and unstable regime, the effects of this slip can disturb the rock for a huge distance depending on volume of cracked rock at depth (preparation focal zone) resulting in anomaly in source zone for precursor signals. Seismological signals for the purposes of the shortterm forecast of earthquakes is practically impossible. Several researchers have attempted earthquake prediction using radon detection techniques in different tectonic environment. Radon serves as an efficient tool in precursory process as the concentration of radon in groundwater does not change much under normal conditions and transport process of radon in different geological setting can be described the basis of physical processes (Papastefanou 2002). The initial investigation on radon presence in groundsoil was performed at an active fault whereby anomalous radon concentrations (Imamura 1947) were reported before the strong earthquake zone (Hatuda 1953).This was there after followed as Tashkhent [Ulomov, 1967] which has been closely followed by a series of radon data observations as anomalies correlated with geophysical events available from different countries. Elaborate research work conducted in Japan (Hirotaka et al., 1988, Igarashi et al., 1995) with Turkey (Inceoz et al., 2006, Friedmann et al., 1988) followed by Mt. Etna, Italy since 2001 (Imme et al., 2005, Morelli et al., 2011). Precursory phenomena of radon in earthquake sequence were observed by (Rastogi et al., 1987) and precursory behavior of helium and radon observed for magnitude 5 earthquakes (Walia et al., 2005). Earthquake occurrence is found to be proportional to the volume of the pre-stressed lithosphere or to the energy of the 
earthquake. The main difference of monitor of VAR from conventional monitoring data of radon of a different type (fixed, numerical values, for example, electrical resistivity, seismic activity, etc.) is that it is not just the amount of VAR at a given point in a given time, and changing the VAR in time. The observed time of earthquake prepare is enough to determine the nature of the behavior of the VAR (Outkin et al., 1999) and, accordingly, the nature of the deformation of the lithospheric block (stretching or compression). Several mechanisms and techniques has been postulated by various investigators to explain the earthquake-related gas anomalies, most involving tectonically induced movement of crustal fluids. Active fault zones suggests that premonitory patterns and the associated earthquakes are possibly incidental results of some broadscale episodic strain changes in the crust (King, 1986). Spatiotemporal monitoring of radon in 70-s - 80-s of the last century (King et al, 1991) in spite of these shortcomings, was the largest scale experiment of radon monitoring, the results of which have yet to be evaluated by researchers while the later monitoring measurement of VAR (Outkin and Yurkov 1997, Outkin,2000) confirmed the fairness of the main conclusions reached after examining the radon monitoring. The observation of anomalies in the radon concentration in soil gas and ground water before earthquakes initiated systematic investigations on earthquake precursor phenomena. Radon concentration variations with changes in flow patterns of diverse fluids within the ground, resultant from modifications in local stress fields of the crust.

\section{Mechanism of radon anomaly for forecasting}

During the experiment (radon monitoring 78-81 anno) in California 25 events with a magnitude of 4.5 to 5.8 occurred. Study of VAR change charts in various locations (total 60 radon monitoring stations) in time for each event showed complete uniformity of conduct VAR in zones of compression or stretching of the array. While these data are corroborated by the simultaneous measurement of conduct VAR for multiple points located in the zones of compression or stretching of the array. Only during the experiment (the period of radon monitoring 78-81anno). The profile of the VAR study at different points of registration (only 60 stations radon monitoring) over time for each event showed complete uniformity of conduct VAR in zones of compression or stretching of the array. In this case, these data support the simultaneous measurement of the behavior of VAR measurements at several points located in areas of compression or stretching of the array. Characteristic changes in the VAR in the preparation and implementation of the earthquake are clearly visible in Figure 1, which shows the spatial and temporal changes of the VAR before the earthquake January 20, 1980 with a magnitude of 5.8. Area of compression and expansion of the array determined by the behavior of the curve in time VAR (Fig. 1, right chart). VAR behavior for zones of compression and tension in the preparation of the earthquake varies. In areas of compression is consistently reducing VAR to some small value, followed by the discharge of the stress state of the array (earthquake). This is evi-

\section{VERSITA}

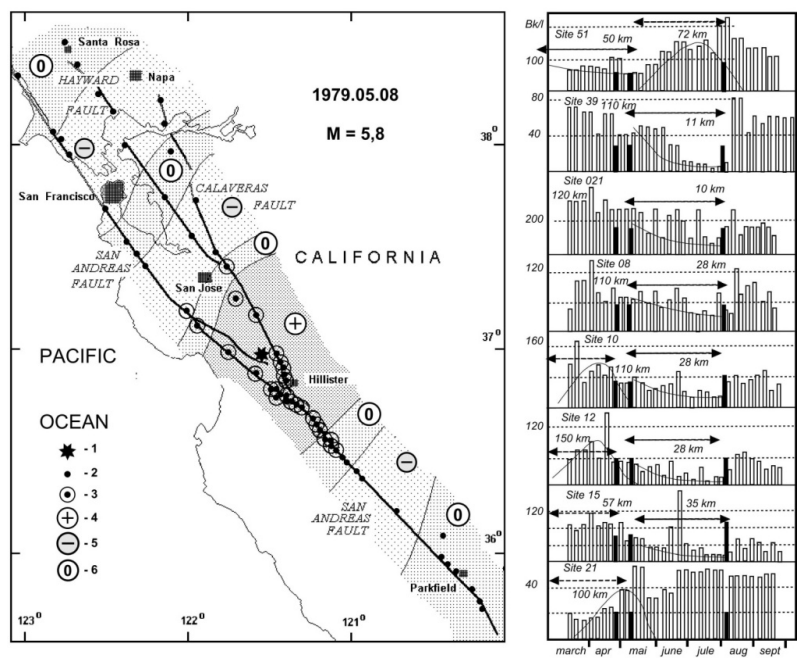

Figure 1. The scheme of spatial and temporal changes in the preparation of VAR earthquake of January 5, 1980 (Utkin 19982000): 1 - The location of the stations recording the UAR, the number of the next station; 2 - Stations selected for analysis of the process of earthquake preparation 3 - the epicenter of the earthquake, 4 - compression zone array, 5 - elongation zone array, 6 - "neutral" zone (a zone with no visible signs of compression or tension).

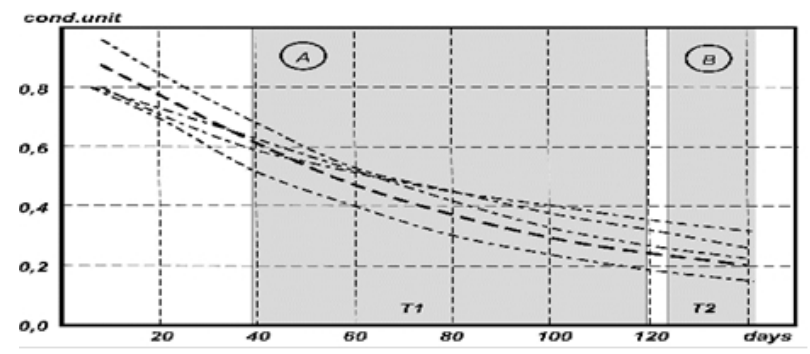

Figure 2. The time variation of radon (VAR) under compression array: A - time T1 - the zone of earthquake preparation B - time T2 - the zone of destruction of the array (tectonic earthquake).

dent from the charts of the VAR for stations $43,46,51,52,58,63$.If radon monitoring station is located in the compression zone of the array, then the change in the concentration of radon in time is an exponential curve of the form:

$$
I_{c}=I_{0}(\exp (-k t))
$$

where $I_{0}$ - some initial VAR intensity before its decline, which lasts from 3 to 5 months (120-150 days); $k$ - compaction ratio of the array (the name of a conditional). Compression process ends the earthquake, destruction (changing) array structure (Fig. 2). It can assume that only the structure interlock friction collapses because from experience know that lithospheric blocks remain unchanged (Rogozhin, 2002). The compression process ends earthquake destruction (change) the structure of the array. We can assume 


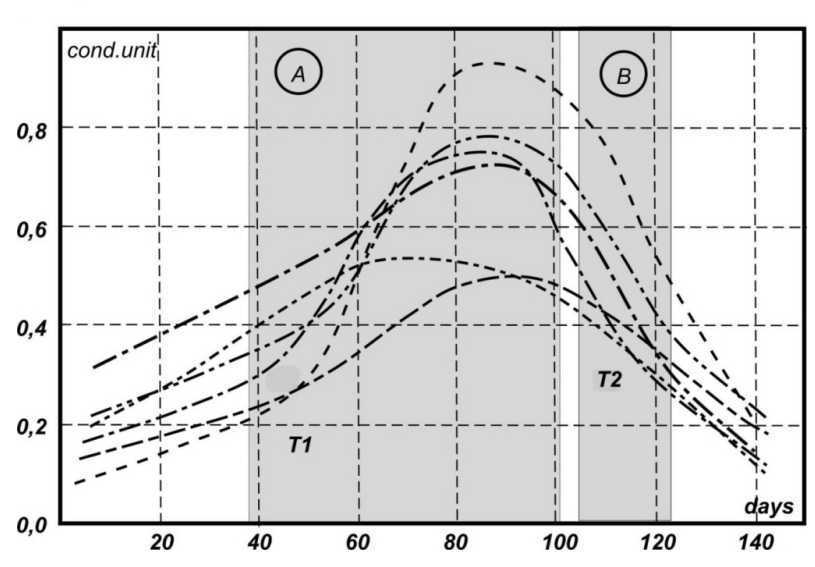

Figure 3. The time variation of radon (VAR) in a stretch of the array: A - duration time T1 zone - the zone of earthquake preparation, T2 - the time zone of destruction of the array (tectonic earthquake).

that only the structure collapses provides interconnect friction, because experience has shown that the lithospheric blocks remain unchanged. The analysis in a mode on-line the data of radonic monitoring, allocation of zones of compression and a stretching of files of researched geodynamic range; allocation of zones of a critical (pre-waste) condition of a file at is intense - deformed condition. Shape of the curve changes VAR compression array and stretch in the array observed at different stations in Fig. 2 and Fig. 3 respectively differ slightly, but for all the curves is possible to distinguish to tell two zones for the time of the earthquake preparation and the reset voltage (actual earthquake).

The last zone is characterized by the almost constant values of the volume activity of radon in time. If radon enters the station location area stretching the array, the change in the concentration of radon (VAR) is more complex (stations 43 and 46 in Figure 1), which is well described by the following

$$
I_{p}=I_{0}(\exp (-k t))
$$

where $I_{0}$ - some initial radon concentration, k.t - magnification radon tensile array where $k$ is the magnification range. VAR curve first increases (increase in radon concentration by stretching the array, increasing its permeability, etc.), reaches a maximum, and then reset the state of stress is observed (the possible collapse of the array), the increase in the array and the decline of radon. Typical curves of the variation of the concentration of radon (VAR) for the various measuring stations in the expansion of the array obtained from experiments monitoring radon (Outkin et al. 1999), are shown in Figure 3. The results presented through work gives concrete proof that the final process of preparation of a tectonic earthquake has a duration of 120-150 days and is not dependent on the type of array deformation (compression or tension) reflection of the process of preparation of a tectonic earthquake in the radon concentrations (VAR) starts for 120-150 days before the event and does not depend on the type of of deformation (compression or a stretching); of the array, whereas the size of area of expected event with magnitude about 4 makes about $40 \mathrm{kms}$; events with magnitude about $50 \mathrm{kms}$ and magnitude 6 more than $50 \mathrm{kms}$. A mathematical modeling of radon concentration anomalies and geodynamical events that shows change of stressed-strained state of the environment in the preparation of the tectonic earthquake (Dutta et al, 2012). Location window after a certain precursor is a key value and should always be given. Perhaps this may be done by introducing a quality factor which is a function of the predicted entrance windows for the quake, the probability and the mean recurrence periods of earthquakes in the considered area. These changes are clearly in VAR curves (Fig. 2 and Fig. 3) received on monitoring data (Outkin and Kozlova 2000). Strain changes get amplified at the earthquake and anomaly sites with sufficient pre-existing stresses that may reach some critical levels (above half fracture strengths) for generation of the earthquakes and anomalies. Energy released in earthquakes is stored along the brittle crust at depths between the surface and $10 \mathrm{~km}$ down; judged from distribution of small earthquakes. Advent of advanced remote sensing methodologies have been initiated to study pre-earthquake deformation detection. A number of researchers are engaged in analyzing relative data collected from different space-borne sensors and groundbased stations to establish a causal relationship between physical mechanisms underlying the earthquake genesis and occurrence and sources of the so-called seismo-electromagnetic emissions. Surface deformation data are provided by GPS and SAR imaging, land surface temperature changes by ASTER, Landsat TM and ETM, different types of cloud studies using MODIS and Seawifs data, electromagnetic and ionosphere anomalies be ground passive stations and radon gas emissions in the faults areas by solid on the ground detectors. Seismologists could then study in real time how strain varies in rocks to identify particular locales where a slip could be about to occur.

\section{Proposed seismic monitoring system}

Based on the study we have proposed an Earthquake alert system consisting of distributed network of remote seismic stations that measure radon count and strong earth motion and transmits the data in real time to a central facility. This facility processes the data and issues warning broadcasts in the form of information packets. Data including space observations are being performed for observing changes in ground elevations as propagating in the surrounding medium allow the earthquake precursors (Zhong and Yue, 2002) to be observed in a finite region of the Earth's surface (precursor area). We have used records for sites 1-20 for File number - number of test wells for year of measurements from 1978 until 1980. For each and every site we calculate the linear trend of the data and average in $\mathrm{km}$ for each variance of radon concentration. There is a slight upward trend to the data. A least squares fit gives the trend line. Applying time series analyses such as fast Fourier transform (FFT), auto-correlation, and cross-correlation, to

\section{VERSITA}


2 year time series data of trace of radon concentration we obtain the station output. The maximum power occurs near frequency $=0.005$ change of concentration of radon for SITE 10 as shown in Fig. $4 a$ ) and $4 b$ showing linear trend of the data and periodogram output for site 10 respectively. It is necessary to know the corresponding station for change of concentration of radon data. The details of the periodogram are calculated for each and every site for 1-20. We record the corresponding stations as output records. From these selected data a scenario to compute daily averages that are not influenced by local sources and sinks of radon. Seasonal cycle and long trend data can be calculated herein and which station can detect an anomaly is determined. Deformations get shifted in time relative to each other indicating propagation of the preseismic strain field from the preparation focal area to the tilt sites, through the rigid blocks of the region. At the onset of the quake there is an electrical signature to the quake, produced by energy released from fracturing rock, that radiates from the quake and which can be sensed by electric potential sensors. When the concentrated stresses in the rock matrix create new micro-cracks and the area and/or volume of pre-existing dilatant volume of cracked rock that drives pore fluids to flow upward through crack network when a vent-like path occurs due to the change of strain leads to dislocation of mineral assemblage in the crystal lattice. This process of dislocation of mineral assemblage is associated with emanation of radon and can be well detected through remote sensing; land based sensors and customized wireless sensor instruments. A specialized earthquake alert system will consist of remote seismic stations to measure radon count change and a central processor and alert algorithms. The central processing facility would then receive two data streams for each station component: one containing radon count,physical parameters updated daily and one containing strong motion data from the accelerometer. Existing seismic monitoring stations require adding a radon count channel. Adding extra bandwidth on the micro seismic station realtime communication channel, a strong motion upgrade can be achieved by adding strong-motion sensors and VCOs: one for each component, and multiplexing these additional data channels on the existing communication channel using a label switch router. Label Switch Router(LSR) is a type of a router located in the middle of a Multiprotocol Label Switching (MPLS) network. When an $L S R$ receives a packet, label included in the packet header as an index to determine the next hop on the Label Switched Path and a corresponding label for the packet from a look-up table. The old label is then removed from the header and replaced with the new label before the packet is routed forward. In normal conditions spare communication bandwidth is not available, switching the labels for data utilization serve as an effective route. The data stream would contain radon flux density and if any anomaly has been observed in two or more than two monitoring stations data the routing of radon data will be labeled in the old routing table. The new packet header will contain data stream that will contain data from the strong-motion sensor till the onset of the earthquake. When

\section{Y \\ VERSITA}

strong motion at the station subsided, the radon count would be passed to the data stream and seismic strong data will be sent as old label.

\section{Results}

Optimum (maximum) number of stations monitoring radon, which is necessary to control the research area can be defined based on the size of the prepare area of tectonic events. This is an area with a radius of $40 \mathrm{~km}$ to earthquakes with magnitudes of about $4 ; 50 \mathrm{~km}$ is radius for earthquakes with $M \geqslant 5$ and a radius of about 50 miles or more for earthquakes with $M \geqslant 6$. Thus, $10-12$ stations radon monitor is enough to monitor the state of lithospheric block of size 200 on $100 \mathrm{~km}$ when controlling the seismic events of magnitude 4. To monitor events with a minimum of magnitude equal to or more than 5 it is enough 6-8 stations to landfill size 100 to $200 \mathrm{~km}$. The complex geological structure study on the number of stations may need to be increased. Obligatory condition of tectonic earthquake is the availability of the required voltage in the Earth's crust, which is called deformation lithological unit or well plates. A sufficient condition for the emergence of some can be considered as external forces that trigger reset voltage when critical levels of stressed-strained state of the array (Outkin et al, 2006). Advantages of geodynamic prior monitoring seismological monitoring consists in the following: (a)monitor of radon is placed directly in the research of radon gas block of rocks, which significantly improves the signal-to-noise ratio when recording data; b) radon monitoring signal allows to judge about the dynamics of stressed-strained state of the block of rock (tensile/compression);c) speed in the dynamics of processes) in preparation for earthquake rocks is relatively small, which allows processing data monitoring in real time. The shortterm prognosis of particularly large earthquakes is entirely based on the monitoring of the Earth's rotation: substantial "triggering function" (variations of the Earth's rotation) shed the accumulated tension over the entire surface of the Earth, causing massive earthquakes. Therefore, warning of major earthquakes must be formed on the basis of monitoring variations in Earth rotation irregularities. The impact of magnitude of earthquake is havoc both in terms of earthquake hazards and breaking of the causative earthquake souce rock with release of tremedous energy as evident from the 2001 Bhuj earthquake (Mw 7.6) (Mishra et al., 2008), and Sumatra Andaman tsunamigenic earthquake (Mw 9.3) (Mishra et al., 2007a, Mishra et al., 2007b). During the earthquake making process, the radon bearing source rock under high stress condition tends to release the gas once the rock matrix started to disintegrate by loosening of the cohesive forces exists among the mineral assemblages of the rock mass when the strain builds up. The process of emanation of radon may be higher for the high asperity zone, which are generally capable to generate strong to great magnitude earthquakes, while the zone of micro tremors $(M<4.0)$ may not be apt for emanating appreciable radon gas before the micro-tremors because of comparatively less competent source rocks that breaks under lesser stress regime in comparison to those of high asperity 

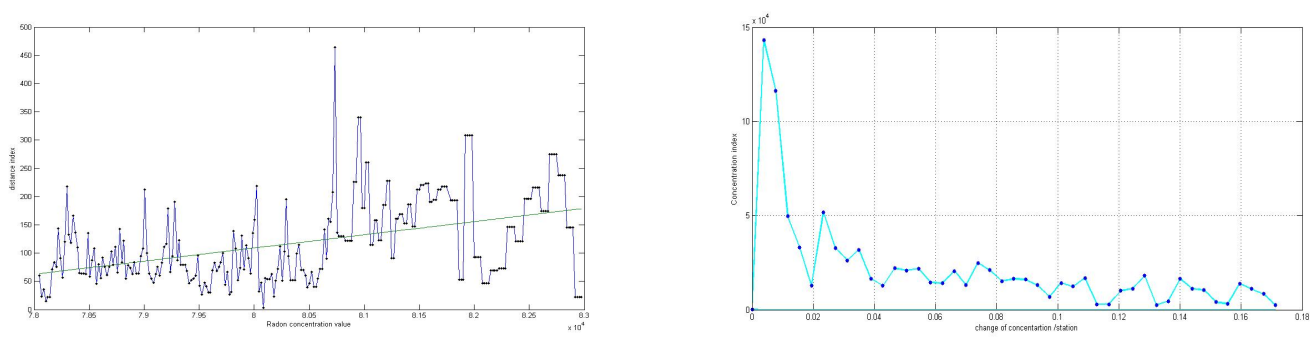

Figure 4.

zones having high stress concentration (Dutta et al., 2012). Such diagnostic behavior of radon emanation during the earthquake making process may be used for earthquake forecast once assessment of the radon emission can be made through its continuous monitoring. Concentration of radon in soil air (in array) hundredths of a percent. Thus, radon is an indicator of fluid movement (solutions) in the array or the soil.

\section{Conclusions}

A method for measuring radon monitoring and aggregation function array speed conventionally named -geodynamic monitoring (GDM) that can be used to solve the problems of short-and medium-term prediction of tectonic earthquakes with minimum use of seismic monitoring data serves the main criteria for operational challenges of forecasting. Some sites suffer increased speed of migration of radon in the researched volume due to convective-dilatancy transport. An algorithm has been already designed whereby two stations which detects anomalies communicate irrespective of other stations in the network for low latency communications of high robustness. A label can be associated to seismic stations which detect anomalies and switch data from seismogram data to radon data transmission is being thought of in future prediction scenarios.

\section{References}

Dutta P. K., Naskar M. K. and Mishra O. P., 2012, Test of strain behavior model with Radon anomaly in earthquake prone zones, Himalayan Geology, Vol. 33 (1), pp. 23-28.

Dutta P. K., Naskar M. K. and Mishra O. P., Test of strain behavior model with radon anomaly: A Bayesian probability approach, Int. J. Geosci., Scientific Research Publishing, doi:10.4236/ijg, 2012.

Friedmann H., Aric K., Gutdeutsch R., King C. Y., Altay C. and Sav H., 1988, Radon measurements for earthquake prediction along the North Anatolian Fault Zone: a progress report. Tectonophysics 152 (3-4), 209- 214.

Hatuda Z., 1953, Radon content and its change in soil air near the ground surface. Memoirs of the College of Science, University of Kyoto, Series B 20, 285-306.

Hirotaka U., Moriuchi H., Takemura Y., Tsuchida H., Fujii I. and Nakamura M., 1988, Anomalously high radon discharge from the Atotsugawa fault prior to the western Nagano Prefecture earthquake ( $m$ 6.8) of September 14,1984 . Tectonophysics 152, 1-2, 147-152.

Igarashi G., Saeki S., Takahata N., Sumikawa K., Tasaka S., Sasaki Y., Takahashi M. and Sano Y., 1995, Ground-water radon anomaly before the Kobe earthquake in Japan Science 269, 60-61.

Imamura G., 1947, Report on the observed variation of the Tochiomata hot spring immediately before the Nagano earthquake of july 15, 1947, Kagaku, 11, 16-17.

Imme G., La Delfa S., Lo Nigro S., Morelli D. and Patane G., 2005, Gas Radon emission related to geodynamic activity of Mt. Etna. Annals of Geophysics, 48, 1, 65-7.

Inceoz M., Baykara, O., Aksoy E. and Dogru M., 2006, Measurements of soil gas radon in active fault systems: a case study along the North and East Anatolian fault systems in Turkey. Radiation Measurements 41, 3, 349-353.

King C.-Y., 1986, Gas geochemistry applied to earthquake prediction: An overview, J. Geophys. Res., 91, 12, 269-12,281.

King Chi-Yu, Walkingstick C. and Basler D., 1991, Field studies of radon in soil, rocks, and water / Wanty R. Gunderson L. and editors / U.S.Geological survey bulletin, pp. 77-133.

Mishra O. P., Zhao D. and Wang Z., 2008, The genesis of the 2001 Bhuj, India, earthquake (Mw 7.6): A Puzzle for Peninsular India, J. Indian Minerals Special Issue, 61, 3 - 4, \& 62, 1- 4, $149-170$

VERSITA 
Mishra O. P., Chakrabortty G. K., Singh O. P., Kayal J. R. and Ghosh D., 2007, Aftershock investigation in the AndamanNicobar Islands: An antidote to public panic, Seismol. Res. Letters., 78, 6, 591-600.

Mishra O. P., Kayal J. R., Chakrabortty G. K., Singh O. P. and Ghosh D., 2007, Aftershock investigation in AndamanNicobar of the 26 December 2004 earthquake (Mw 9.3) and its seismotectonic implications, Bull. Seismol. Soc. Am., 97, 1A, S71-S85.

Morelli D., Imme G., Altamore I., Cammisa S., Giammanco S., La Delfa S., Mangano G., Neri M. and Patane G., Radionuclide measurements, via different methodologies, as tool for geophysical studies on Mt. Etna. Nuclear Instruments and Methods in Physics Research A, DOI NIMA 10.1016/j.nima.2011.01.172,2011.

Outkin V. I. and Yurkov A. K., 1997, Radon and tectonic earthquakes problem Vulcanology and seismology, 4, pp. 84-92.

Outkin V. I. and Yurkov A. K., 1997, Reflection seismic events in the radon exhalation Geophysics, m.: EAGO, 6, pp. 50-56.

Outkin V. I., Kozlova I. A. and Yurkov A. K., 1999, Radon as an indicator of stress state array Mel/nikovskie read the book perm: Mining Institute UB RAS, pp. 79-82.

Outkin V. I., 2000, Spatio-temporal monitoring of radon and the problem of the medium -term forecast of tectonic earthquakes//Ural geophysical journal, no. 1, Yekaterinburg: IGF UB RAS, pp. 101-106.

Outkin V. I., Mamyrov E., Kang M., Krivasheev S. V., Yurkov A. K., Kosyakin I. I. and Shishkanov A. N., 2006, Radon Monitoring study of preparation process of tectonic earthquake on the Northern Tien Shan.Physics of the Earth, 9, pp. 145-155.

Outkin V. I. and Kozlova I. A. 2000, Massif was straining the reflection in the dynamics condition radon field / / XXV General Assembly EGS, Nica, France, p. 1356-1358.

Outkin V. I., Yurkov A. K., Klimshin A. V. and Kozlova I. A., 2011, Geodynmic monitoring in real times. AGU 2011,1203407, USGS, S-Franc., Institute of Geophysics.

Papastefanou C., 2002, An overview of instrumentation for measuring radon in soil gas and groundwaters. J Environ Radioact. 63, 3, 271-283.

Rastogi B. K., Chadha R. K. and Raju I. P., 1986, Seismicity near Bhatsa reservoir, Maharashtra, India. Physics of the Earth and Planetary Interiors 44, 2, 179-199.

Rogozhin E. A., 2000, History intensified seismogenerating structures of Northern Eurasia in the Holocene//Rep. RAS, 370, 3, pp. 390-392.

Sarson G. R. and Jones C. A., 1999, A convection driven geodynamo reversal model; Physics of the Earth and Planetary Interiors 111, pp-3-20.

Ulomov V. I., Zakharovc A. I. and Ulomova N.V., 1967, Tashkent earthquake of April 26, 1966, and its aftershocks. Akad Nauk SSSR, Geophysic 177, 567-570.

Walia V., Virk H. S. and Bajwa B. S., 2006, Radon Precursory Signals for Some Earthquakes of Magnitude $>5$ Occurred in N-W Himalaya: An Overview Pure \& Applied Geophysics;Apr2006, $163,4, \mathrm{p} 711$.

Zhong L. and Yue L. S., 2002, Earthquake Precursor forecasting system based on GIS [M]. Chengdu Map Press. 